Article

\title{
Optimal Design of Cogeneration Systems in Industrial Plants Combined with District Heating/Cooling and Underground Thermal Energy Storage
}

\author{
Andrea Reverberi, Adriana Del Borghi and Vincenzo Dovì * \\ Department of Chemical and Process Engineering, University of Genoa, Via Opera Pia 15, \\ Genova 16145, Italy; E-Mails: reverb@dichep.unige.it (A.R.); adry@unige.it (A.D.B.) \\ * Author to whom correspondence should be addressed; E-Mail: dovi@istic.unige.it; \\ Tel.: +39-010-3532921; Fax: +39-010-3532586.
}

Received: 2 November 2011; in revised form: 28 November 2011 / Accepted: 2 December 2011 / Published: 6 December 2011

\begin{abstract}
Combined heat and power (CHP) systems in both power stations and large plants are becoming one of the most important tools for reducing energy requirements and consequently the overall carbon footprint of fundamental industrial activities. While power stations employ topping cycles where the heat rejected from the cycle is supplied to domestic and industrial consumers, the plants that produce surplus heat can utilise bottoming cycles to generate electrical power. Traditionally the waste heat available at high temperatures was used to generate electrical power, whereas energy at lower temperatures was either released to the environment or used for commercial or domestic heating. However the introduction of new engines, such as the ones using the organic Rankine cycle, capable of employing condensing temperatures very close to the ambient temperature, has made the generation of electrical power at low temperatures also convenient. On the other hand, district heating is becoming more and more significant since it has been extended to include cooling in the warm months and underground storage of thermal energy to cope with variable demand. These developments imply that electric power generation and district heating/cooling may become alternative and not complementary solutions for waste energy of industrial plants. Therefore the overall energy management requires the introduction of an optimisation algorithm to select the best strategy. In this paper we propose an algorithm for the minimisation of a suitable cost function, for any given variable heat demand from commercial and domestic users, with respect to all independent variables, i.e., temperatures and flowrates of warm fluid streams
\end{abstract}


leaving the plants and volume and nature of underground storage. The results of the preliminary process integration analysis based on pinch technology are used in this algorithm to provide bounds on the values of temperatures.

Keywords: surplus process heat; cogeneration; district heating/cooling; optimal apportionment

\section{Introduction}

The increasing cost of energy and the continual sharpening of carbon dioxide emission standards in the last decades have urged companies to implement energy saving measures in the design of new industrial processes and when retrofitting existing plants. The most significant savings have been obtained through the energy integration of systems of gradually increasing size.

Energy integration inside a process is now a well-established, mature technology, mainly based on exergy methods and pinch analysis. Similarly, energy integration across different processes (the so-called total site integration) has led to considerable advances in the overall efficiency by optimally combining energy needs and availability [1-3]. The inclusion of a bottoming cycle to produce electrical or mechanical shaft power has been part of this integration strategy for some time now, as well as the use of waste heat at lower temperatures for commercial and/or domestic heating. Furthermore, the introduction of new engines, such as the ones using the organic Rankine cycle, capable of employing condensing temperatures very close to the ambient temperature, has made the generation of electrical power at low temperatures also convenient [4].

However, this may enter into conflict with the use of lower temperature energy for district heating, which is becoming more and more significant since it has been extended to include cooling in the warm months and underground storage of thermal energy to cope with variable demand. Thus, district heating/cooling has become a key issue in territorial energy planning. Large power stations have had a pioneering role in the past and are still at the forefront of this area. However, market pressure and the need to comply with more and more stringent directives and guidelines have encouraged mid to large industrial plants, as well as farming enterprises, to consider integration with the surrounding territory. Regional energy integration which includes the optimal integration of industrial, agricultural, municipal and domestic energy sources and demands over a limited area, is presently in an advanced stage of development. Feasibility analyses are routinely carried out and actual implementations have been made wherever a general agreement has been reached among all the stakeholders involved.

The presence of alternative options (electrical power or heat available for territorial energy planning) requires the introduction of suitable optimisation algorithms to select the optimal strategy. In this article we examine the options available to both managers and design engineers of industrial plants for the selection of the optimal energy management when territorial integration is taken into account. To this purpose we present an optimisation algorithm that considers possible scenarios and the constraints they are subject to for the attainment of technically sound and financially feasible solutions. 


\section{Results and Discussion}

\subsection{Energy Sources in Industrial Plants}

Potential heat sources are all the streams of the process considered that must be cooled. The heat removed from these streams is first used, whenever possible, inside the process to heat other streams which undergo a phase change or whose temperature has to be increased. This heat transfer is obviously limited by the condition that the temperatures of the streams losing energy be greater than the temperatures of the streams to which the energy is transferred.

A powerful implementation of this fundamental principle in process engineering is the well known and widely applied pinch technology [5]. The overall heat requirement is first calculated by plotting the thermal loads of the cold streams on a single graph (cold composite curve) in a temperature - enthalpy plane. If the specific heats remain constant in the temperature range of each stream, the resulting graph is a piecewise linear function with a slope equal to the inverse of the heat capacity of each stream or of a combination of them if temperature ranges overlap. Similarly, the thermal loads of the hot streams (i.e., the streams that must be cooled) can be plotted in a similar graph to provide the hot composite curve. The optimal heat integration can be identified by letting the minimum temperature difference between the curves be equal to a suitably pre-determined $\Delta T$ (Figure 1).

Figure 1. Hot and cold composite curves of a generic process.

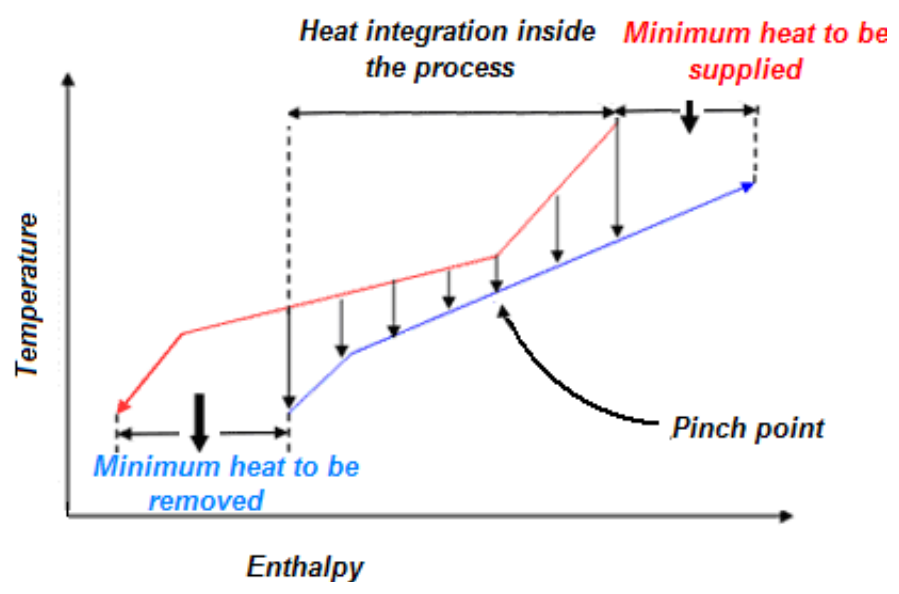

The corresponding temperature is called pinch temperature and the fundamental law of pinch technology is the requirement that no heat transfer take place across the pinch. Any such transfer would increase by the same amount both the overall heating and cooling requirement of the process. The grand composite curve can be constructed by plotting enthalpy differences between the composite curves at each temperature (Figure 2).

The pinch point lies now on the temperature axis and temperature intervals in which hot streams lie above cold streams can be used for the heat integration of the process. The overall minimum thermal requirement and the minimum required amount of cooling water of the process can now be easily identified as the abscissas of the curve at maximum and minimum temperatures. 
Figure 2. Grand composite curve of a generic process.

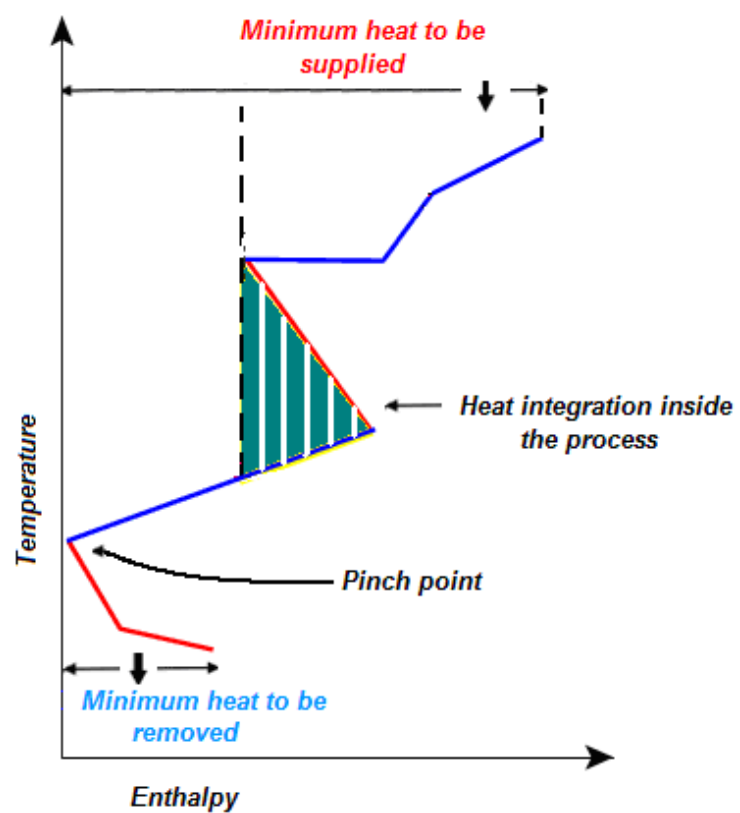

In addition to providing this information, the grand composite curve is a powerful tool that experienced process designers can take advantage of to optimally modify the configuration of the plant. For instance, in Figure 3 optimal process integration makes it possible to use only low pressure steam, whereas the heat necessary at higher temperatures is provided by internal energy transfer.

Figure 3. Process integration makes it possible to use low pressure steam only.

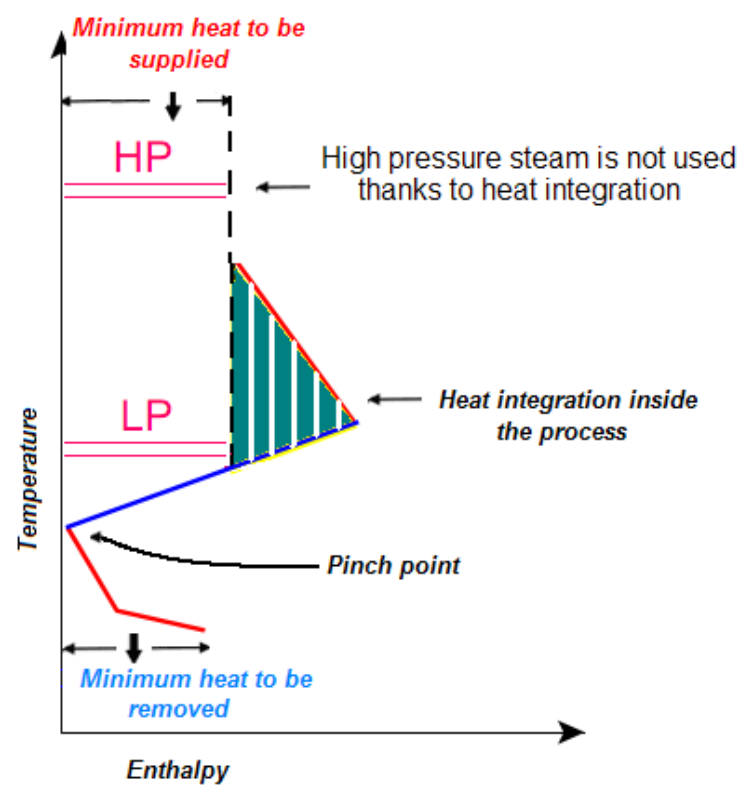

Similarly in Figure 4 only part of the heat is provided by high pressure steam. By considering heat requirements at different temperatures the energy transfer is optimally split between the two steam lines.

In Figure 5 the use of internal energy transfer and the recovery of the enthalpy content of flue gases makes it possible to dispense with external energy sources. Indeed the flue gas temperature $v s$. 
enthalpy curve (which is a straight line if the specific heat is approximately constant) lies entirely above the grand composite curve of the process and consequently it can supply all the necessary amount of heat.

Figure 4. Reduced use of high pressure steam.

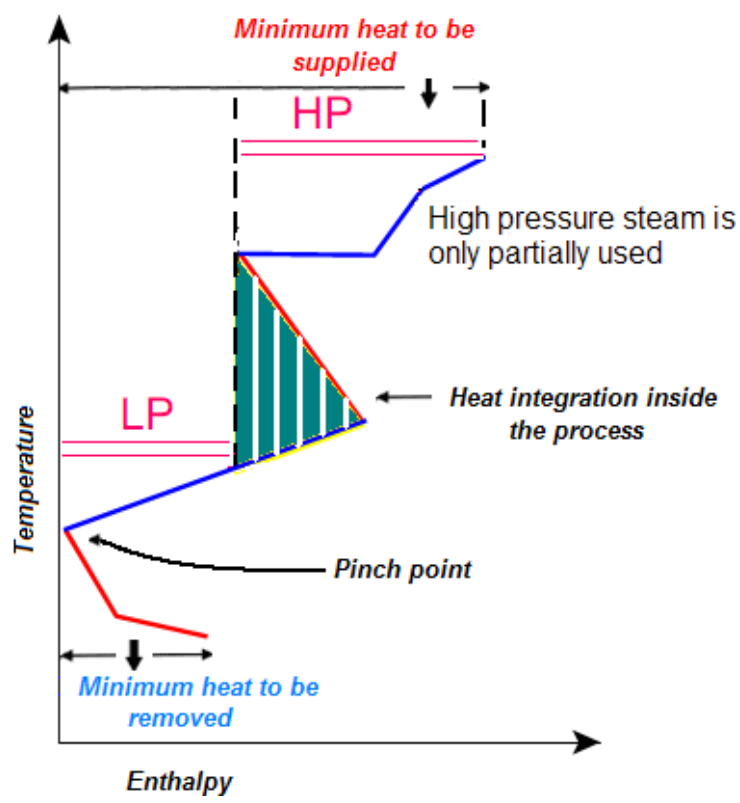

Figure 5. Recovery of flue gas enthalpy content.

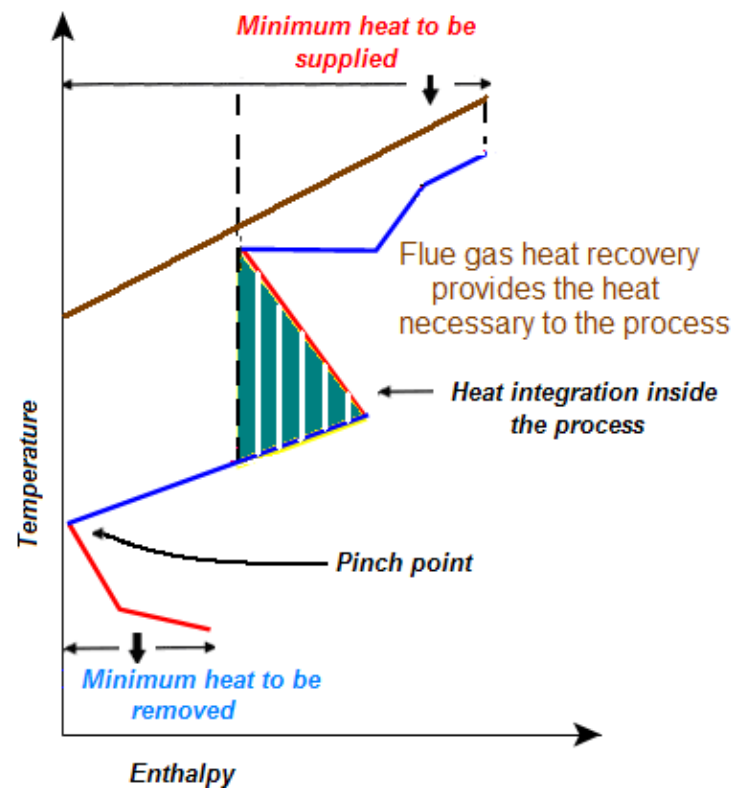

Sometimes external work may be a convenient way to raise the temperature at which heat is available. This is often the case when large amounts of heat are available just below the pinch temperature and corresponding amounts of energy are required at temperatures slightly superior to the pinch temperature (Figure 6). In these cases the cost of using the work of heat pumps can be less (and sometimes much less) than the cost of the amount of heat saved. Obviously the use of available energy inside the process through heat integration has a higher priority than exporting energy outside of it. 
Thus, the identification of potential heat sources in the design phase or in a retrofitting process should not consider the energy that might be conveniently used in a process integration analysis.

Similarly, total site integration (i.e., energy integration between different processes located at the same industrial site) can be considered a straightforward extension of the heat integration in a single process. Indeed, with respect to district heating/cooling it has the obvious advantage of a limited infrastructural effort.

Figure 6. Use of a heat pump to exploit heat available below the pinch.

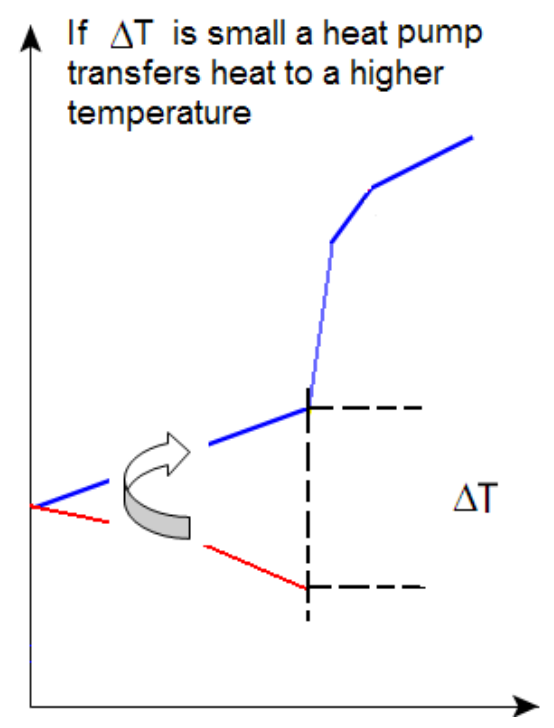

Once both process and site heat integration has been carried out, the amount of energy available "across the fence" can easily be determined by simple inspection.

Below the pinch this can be expressed typically in the form:

$$
0 \leq \dot{E}_{i} \leq \sum_{k} c_{p_{k}} F_{k}\left(T_{\max _{i k}}-T_{\min _{i k}}\right) \quad i=1, \ldots, N \quad k=1, \ldots, M
$$

where $\dot{E}_{i}$ is the power available in the temperature range:

$$
\max _{k}\left(T_{\max _{i k}}\right)-\min _{k}\left(T_{\min _{i k}}\right) \quad k=1, \ldots, M
$$

and $c_{p k}$ the average specific heat of the flowrates $F_{k}$.

These constraints apply to all the temperature intervals $N$, where an energy export is available. Above the pinch the amount of energy available that can be transferred depends on how heat is supplied to the process. The excess heat can be recovered either generating shaft work in a turbine or exchanging heat with external fluids.

Thus, for instance, in Figure 7 the amount of energy available when heat is supplied using the flue gases resulting from fuel combustion is shown. In this case part of the energy required by the process can be recovered through heat integration inside the process and consequently the flue gas can provide an amount of surplus heat from the combustion temperature to the temperature where energy transfer to the process is required. 
Figure 7. Determination of available heat when heat is supplied from fuel combustion.

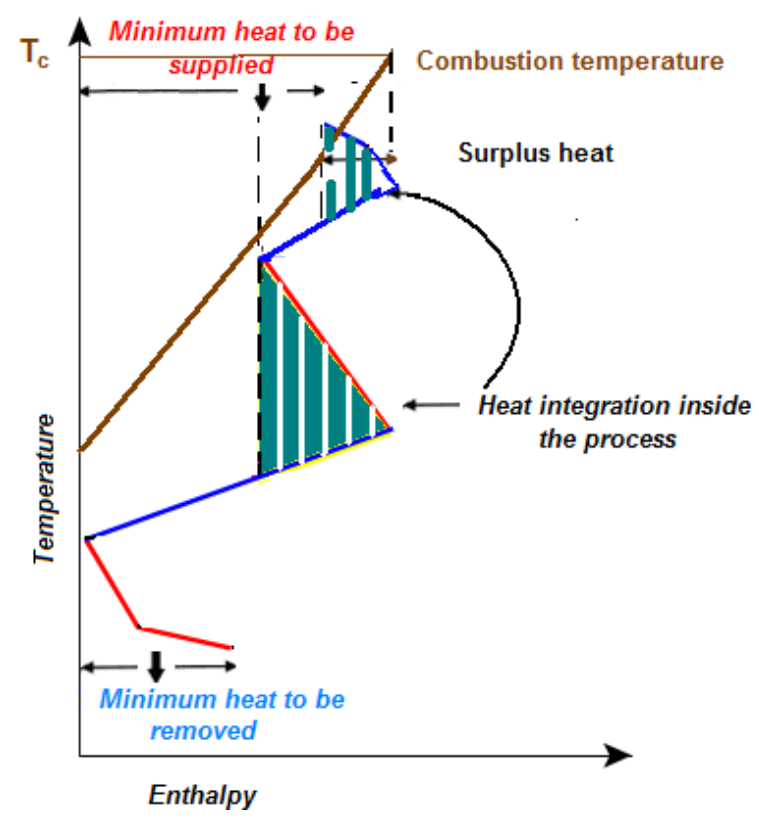

\subsection{Energy Transfer Options}

\subsubsection{Electrical Power}

In industrial processes cogeneration (or combined heat and power generation, $\mathrm{CHP}$ ) means production of electrical power by gas turbines (using flue gas resulting from fuel combustion, Figure 7) or by steam turbines operating in a Rankine cycle using the available surplus heat. This is the main difference with large power stations where heat is a byproduct of power generation and gas turbines or reciprocating engines are normally used.

The key information provided by the pinch analysis is that the temperatures over which the Rankine cycle operates should not include the pinch point. Thus, the first possibility is provided by a Rankine cycle operating between two temperatures above the pinch temperature. This situation can arise when high pressure steam has to heat up a cold stream over a wide temperature range. As shown in Figure 8 part of the high pressure steam can be fed to a turbine whose exhaust steam temperature is still high enough to provide the necessary heat to the lower temperature part of the stream.

Whenever the pinch temperature is high enough (as is the case for instance in the ammonia production process), a CHP system can be designed for cooling the hot streams without the necessity of using a coolant, which adds to the profitability of the process. Again a steam-operated Rankine engine is used to this purpose. The steam generated by the heat transferred from the process is the working fluid that powers the steam turbine.

If an Organic Rankine Cycle (ORC) is used, it is possible to recover heat from low temperature sources, thanks to the use of an organic fluid, which has a boiling point, at which the liquid-vapor phase change takes place, lower than the traditional water-steam system. This greatly increases the number of processes to which it can be applied. 
Figure 8. Cogeneration above and below the pinch.

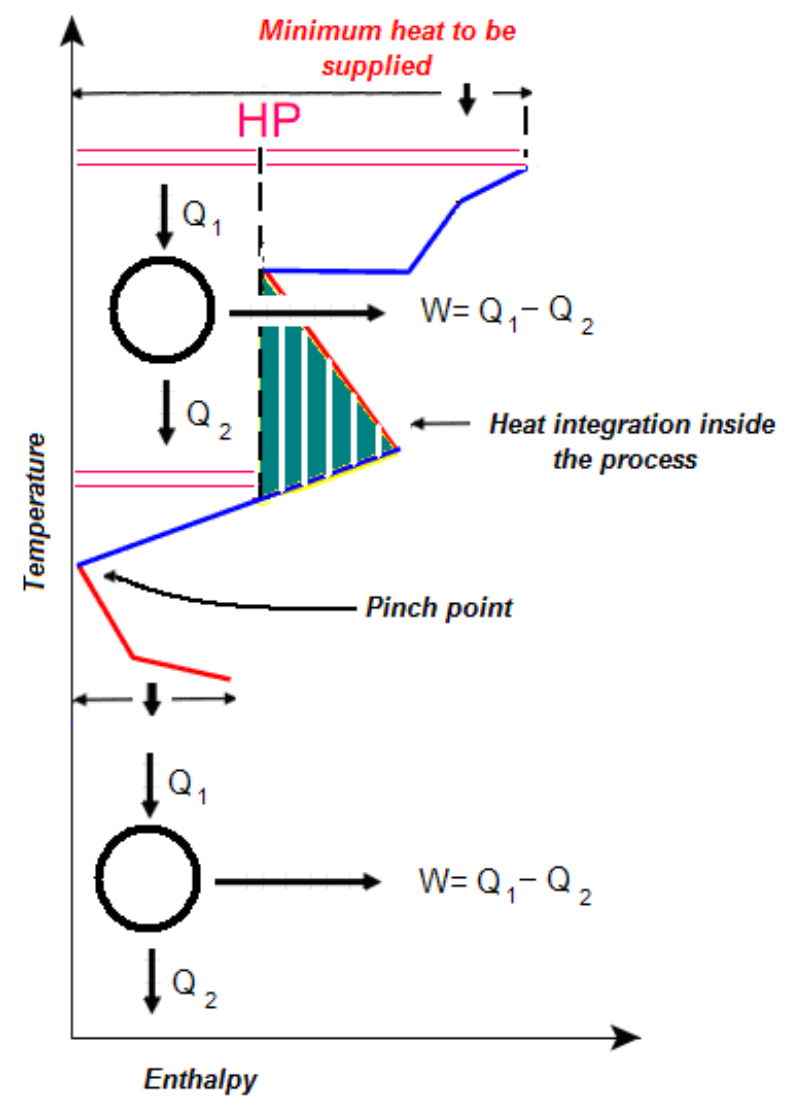

Thus, the temperature range over which the combined heat and power generation is possible frequently overlaps the range traditionally used for district heating/cooling. Hence the necessity of developing an optimisation algorithm for the most convenient distribution of waste heat from processes between the two options.

\subsubsection{District Heating and Cooling}

Water is the most frequently used medium to distribute heat collected from various sources to dwellings and offices through heat substations, although steam is sometimes used [6]. As anticipated before, an intermediate storage greatly adds to the profitability of the system.

The size of district heating systems varies from covering large urban areas to small villages and even to a limited number of households. According to the size of the system there can be, in addition to the primary radial or ring-shaped piping networks, secondary and tertiary circuits with decreasing pipe diameter, whose shape and size is adapted to the urban structure served by the system.

In addition to the recovery of surplus heat which otherwise would be released to the environment, district heating reduces the investment costs for domestic or commercial heating equipment. However, this cost reduction is more than offset by the capital costs for the construction of district heating networks. Therefore areas with a low population density or small building compounds are generally not suitable for district heating, as investment costs per household would be considerably high.

The energy generated or stored can be used also for cooling purposes, i.e., for providing chilled water to households and commercial space. The change from a district heating to a district 
heating/cooling system may guarantee the affordability and financial sustainability of a large scale project, in that both storage capacity and electrical power are considerably reduced [7]. Indeed the seasonal heat storage is limited to the autumn months for the heating period and to spring for the cooling time, as opposite to a spring/summer/autumn storage time in the purely heating scheme. Obviously the storage capacity is reduced correspondingly.

Similarly electricity consumption is greatly reduced if heat driven refrigerators (such as absorption refrigerators, [8]) are used, instead of the traditional compressor refrigerators. Up to $90 \%$ electricity consumption reduction can be achieved in this case.

\subsection{The Underground Thermal Energy Storage}

The installation of intermediate heat storage on a seasonal basis greatly adds to the overall profitability of a district heating system, as the energy produced in periods of limited consumption is not dispersed to the environment. Additionally, it can even out variable energy demands. However, it requires larger volumes than storages used for intermittent renewable sources based on a day/night or windy/calm weather cycle. Obviously, larger containers give rise to considerable investment costs, which play a key role in the feasibility study and cost/benefit analysis of a district heating/cooling project.

Considerable savings can be attained by storing the available heat using a natural medium. With respect to the construction of large steel or concrete reservoirs (above or partially below ground), underground thermal energy storage (frequently referred to with the acronym UTES) can reduce the payback time of capital costs to approximately 5-10 years [9].

Indeed large quantities of heat can be stored in underground geological structures such as aquifers (ATES), natural or artificial caverns and disused mines (CTES) or drilling borehole thermal energy storage structures (BTES).

Occasionally, the two technologies are combined: a high temperature storage vessel embedded in an underground structure, which is kept at a lower temperature and from which heat is extracted using heat pumps. This makes it possible to recapture some of the heat released by the central vessel to the external environment.

Indeed heat losses to the environment depend heavily on temperature, size and nature of the storage system considered. This has to be taken into account in the general optimisation procedure by considering different options separately. Alternatively, a preliminary optimisation of suitably identified subsystems can be carried out with respect to a limited number of parameters, which are then kept fixed at their optimum values in the general optimisation procedure.

Thus for instance heat-driven cooling can be either dispersed or decentralised [10] according to whether the chilled water used for cooling is generated by small chillers located in each user's building (thus dispensing with chilled water distribution) or through small cooling substations based on absorption technology (thus reducing chilled water distribution costs and eliminating the necessity of installing chillers in each building). Once the better solution for the particular case considered has been identified, it can be used in the general optimisation procedure. 


\subsection{Model for the Identification of the Optimal Configuration}

The overall optimal configuration can be evaluated if a suitable objective function is defined subject to the constraints. Typically the objective function depends on the revenue from energy transferred outside the plant and on both capital and operating costs related to the energy transfer process.

Each heat source $i(i=1, \ldots, N)$ not used inside the process can be used either for power generation or for district heating/cooling. The number of heat sources is equal to the number of temperature ranges over which heat is available. For large temperature ranges more than one heat source can be considered.

The annual revenue for each of them is given by $b_{1 i} \cdot\left(\varepsilon_{i} \cdot c-m_{1 i}\right)+b_{2 i} \cdot\left(H_{i} \cdot d-m_{2 i}\right)$ where $b_{i}$ is the binary value $\{0,1\}, \varepsilon_{i}$ and $H_{i}$ the yearly amounts of power generated or of heat transferred to a district heating system in one year and $c$ and $d$ their prices. $m_{1 i}$ and $m_{2 i}$ are operating and maintenance costs of the equipment operating in the interval $i$. The product $b_{1 i} \cdot b_{2 i}$ must obviously be equal to zero (the available energy is used for electrical work, for heat transfer or released to the environment, the latter condition being given by the condition that both $b_{1 i}$ and $b_{2 i}$ are zero.

The capital costs are the turbine/generator equipment for the cogeneration and heat exchangers and volume and nature of the underground storage for the district heating. The annual fixed charges $f$ can be computed by dividing the capital costs by the total years of useful life (supposing salvage values equal to zero).

Thus a reasonable objective function is:

$$
\Phi=-\sum_{i}\left[b_{1 i} \cdot\left(\varepsilon_{i} \cdot c-m_{1 i}-f_{1 i}\right)+b_{2 i} \cdot\left(H_{i} \cdot d-m_{2 i}-f_{2 i}\right)\right]
$$

subject to:

$$
b_{1 i} \cdot b_{2 i}=0 \text { for all } i
$$

which is to be minimised subject to the mass and heat balances that must be taken into account in the overall system. Both $\varepsilon_{i}$ and $H_{i}$ are equal to $\dot{E}_{i}$ to a factor of proportionality for changing $\mathrm{kW}$ to $\mathrm{kWh} /$ year. This is not just a conversion factor, as it takes into account the number of operating hours in a year.

Obviously additional factors could be introduced into the objective function. Typically they might include terms related to robustness (with a view to minimising the number of faults of the system) and to resilience (so that the overall system can adapt to changes in exogenous variables, while remaining technically stable and financially feasible).

Additional equality constraints are provided by the energy and mass conservation law. The energy transfer balance between the process and the district heating system is given by (see Figure 9):

$$
\begin{aligned}
& \sum_{i} b_{2 i} \dot{E}_{i}=\bar{c}_{p} \rho q_{1}(t)\left(T_{1}(t)-T_{0}\right) \\
& T_{1}(t) \leq \max _{i}\left(b_{2 i} T_{\max , i}\right)
\end{aligned}
$$


Figure 9. Conceptual scheme of a district heating network with an intermediate storage.

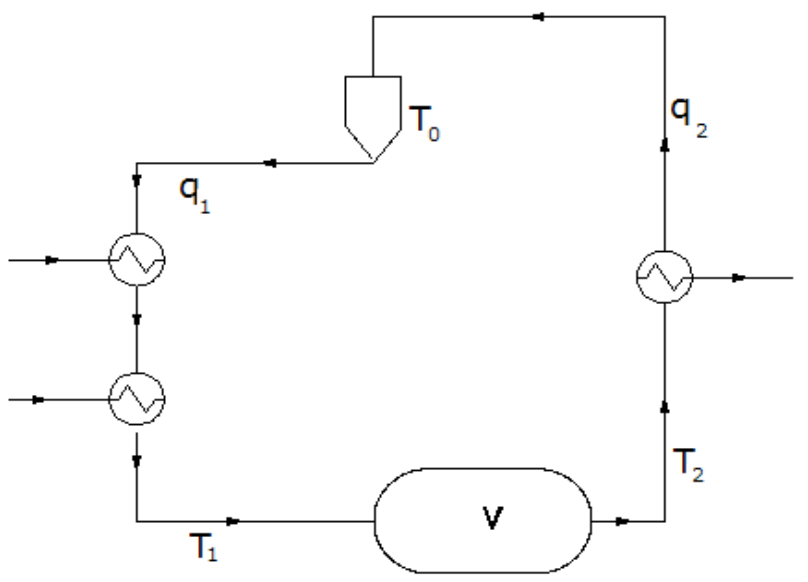

Similarly the energy transfer between the district heating system and the final user is given by:

$$
\bar{c}_{p} \rho q_{2}(t)\left(T_{u}(t)-T_{0}\right)=\dot{Q}(t)
$$

where $\dot{Q}(t)$ is the heat demand from final users (which has to be estimated on basis of historical demand values) and $T_{u}(t)$ is the temperature of the underground storage.

The mass and energy balances in the underground storage can be expressed as:

$$
\begin{aligned}
\frac{d V}{d t} & =q_{1}(t)-q_{2}(t) \\
V(0) & =V_{0} \\
V \frac{d T_{u}}{d t} & =q_{1}(t) T_{1}(t)-q_{2}(t) T_{u}(t) \\
T_{u}(0) & =T_{0}
\end{aligned}
$$

The minimisation has to be carried out with respect to the set $\left\{q_{1}(t)\right\} \otimes\left\{b_{k i} \mid k=1,2 ; i=1, . ., N\right\}$ over the span of one year. For every value of $\left\{q_{1}(t)\right\} \otimes\left\{b_{k i} \mid k=1,2 ; i=1, . ., N\right\}, T_{1}(t)$ can be calculated from Equation (2), $q_{2}(t)$ from Equation (3), $V(t)$ from the integration of Equation (4) and $T_{u}(t)$ from the integration of Equation (5).

Once the minimisation has been carried out, the optimal values of $b_{k i}\left\{\hat{b}_{k i}\right\}$ and the maximum value of $V(t)$ found in the time range considered provide all the information necessary to carry out the design of the cogeneration equipment and of the district heating/cooling system, including the volume of the underground thermal energy storage.

Obviously in case the minimum value of $\Phi$ should turn out to be positive, the investment costs would exceed the financial benefits: the project would not be profitable and would not be implemented.

\subsection{Numerical Algorithm for the Solution of the Optimisation Problem}

The function $q_{1}(t)$ can be parametrised in terms of a finite number of parameters, the simplest approximation being a piecewise constant function:

$$
q_{1}(t)=\bar{q}_{1, j} \quad \text { if } t_{j} \leq t \leq t_{j+1} \quad j=1, \ldots, T P
$$


Figure 10. Flow chart of the optimisation algorithm.

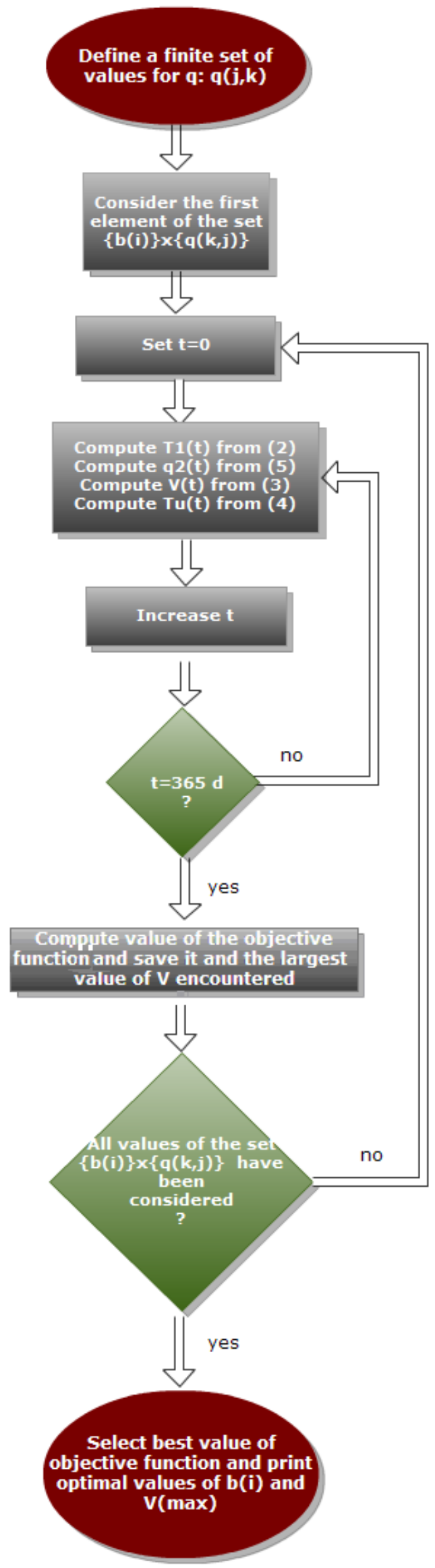


The number of intervals can be set equal to the number of periods in the year during which $\dot{Q}(t)$ is approximately constant. Thus the space of independent variables $\left\{q_{1}(t)\right\} \otimes\left\{b_{k i} \mid k=1,2 ; i=1, . ., N\right\}$ is changed into the finite dimensional space $\left\{\bar{q}_{1, j}\right\} \otimes\left\{b_{k i}\right\}$.

The optimization with respect to $\left\{b_{i}\right\}$ is carried out by enumeration, i.e., all the combinations are considered and the minimisation is carried out with respect to the set $\left\{\bar{q}_{1, j}\right\}$. The best combination is then considered for the determination of $\left\{b_{i k}\right\}$.

While the minimisation of (1) with respect to the set $\left\{\bar{q}_{1, j}\right\}$ with fixed values of $\left\{b_{i k}\right\}$ can in principle be carried out using rigorous gradient methods, considering GP equally spaced values of $\bar{q}_{1, j}$ over a convenient grid (i.e., $\left.\left\{\bar{q}_{1, j r} \mid r=1, \ldots, G P, k=1, \ldots, T P\right\}\right)$ is a reasonable approximation which greatly simplifies the optimisation task. Additionally convergence to a sub-optimal local minimum (which might occur if a gradient method is used) can be avoided.

In other words, the solution of Equations (2-5) is carried out for each element of the $\left(2^{N} \times T P \times G P\right)$-dimensional set $\left\{\bar{q}_{1, j r}\right\} \otimes\left\{b_{i k}\right\}$ and the objective function is computed for each solution. Again the combination of values that provide the best value of the objective function is the sought after solution of the optimisation problem. The flow-chart of the minimisation procedure is shown in Figure 10. For the sake of illustration simplicity energy losses have not been considered. However, they can easily be introduced by suitably modifying the energy balances (2), (3), (5). As mentioned in Section 3, different types of heat storage can have different expressions for leaks and losses. In this case too, the procedure has to be repeated for each scenario and the corresponding values of the objective function should be examined for comparison. Obviously this increases further the enumeration effort. However, due to the computational efficiency with which the system of Equations (2-5) can be solved, the overall optimum can be located with an affordable computational time.

\section{Conclusions}

Surplus and waste heat from industrial plants is becoming an important issue in the overall strategy for the reduction of the global carbon footprint. While the energy efficiency of single processes is now a mature technology and heat integration at industrial site level has already attained significant results, the territorial energy integration including the key aspects (i.e., energy sources, energy consumption and intermediate energy storage) is still in a development phase.

This is partly due to the large number of options available, such as the availability of several different energy sources, the possibility of using the energy in a variety of ways for different purposes and the existence of suitable natural energy storages.

The presence of several alternatives requires the introduction of suitable optimisation algorithms capable to select the most convenient technical solution. This implies the identification of an adequate objective function and of an accurate model describing the relations among the physical variables.

To this end we have proposed a model for the selection of the optimal apportionment of process surplus heat between electrical work and district heating/cooling when seasonal thermal energy storage is available. The model is based on well established techniques of process integration for the identification of energy sources and on mass and energy conservation laws outside the process. 


\section{Notation}

$b_{1 i} \quad$ Binary value $\{0,1\}$ to indicate use of waste energy for electrical power generation in interval $i$

$b_{2 i} \quad$ Binary value $\{0,1\}$ to indicate use of waste energy for heat transfer in interval $i$

c Unit price of electricity $(\$ / \mathrm{kWh})$

$c_{p k} \quad$ Specific heat $\left(\mathrm{kWh} / \mathrm{kg}{ }^{\circ} \mathrm{C}\right)$ of stream $k$

$\bar{c}_{p} \quad$ Specific heat of medium in district heating $\left(\mathrm{kWh} / \mathrm{kg}{ }^{\circ} \mathrm{C}\right)$

$d \quad$ Unit price of electricity $(\$ / \mathrm{kWh})$

$\dot{E}_{i} \quad$ Rate of energy transfer in interval $i(\mathrm{~kW})$

$f_{i} \quad$ Annual fixed charges (\$/year)

$F \quad$ Flowrate of stream $\mathrm{k}(\mathrm{kg} / \mathrm{h})$

$H_{i} \quad$ Amount of heat transferred to a district heating system per year from interval $i(\mathrm{kWh} / \mathrm{year})$

$m_{i} \quad$ Annual costs for operation and maintenance (\$/year)

$q_{1} \quad$ Flowrate of medium fed to intermediate storage $\left(\mathrm{m}^{3} / \mathrm{h}\right)$

$q_{2} \quad$ Flowrate of medium leaving intermediate storage $\left(\mathrm{m}^{3} / \mathrm{h}\right)$

$\dot{Q} \quad$ Heat demand $(\mathrm{kW})$

$T$ Temperature $\left({ }^{\circ} \mathrm{C}\right)$

$T_{0} \quad$ Ambient temperature $\left({ }^{\circ} \mathrm{C}\right)$

$T_{u} \quad$ Temperature of intermediate storage $\left({ }^{\circ} \mathrm{C}\right)$

$V \quad$ Volume of intermediate storage $\left(\mathrm{m}^{3}\right)$

$\varepsilon \quad$ Amount of electrical power generated per year from interval $i(\mathrm{kWh} /$ year $)$

$\rho \quad$ Density of heating medium $\left(\mathrm{kg} / \mathrm{m}^{3}\right)$

$\Phi \quad$ Objective function

\section{References and Notes}

1. Linnhoff, B.; Townsend, D.W.; Boland, D.; Hewitt, G.F.; Thomas, B.E.A.; Guy, A.R.; Marsland, R.H. A User Guide on Process Integration for the Efficient Use of Energy; Elsevier: Amsterdam, The Netherlands, 1982.

2. Klemeš, J.; Dhole, V.R.; Raissi, K.; Perry, S.J.; Puigjaner, L. Targeting and design methodology for reduction of fuel, power and CO2 on total sites. Appl. Therm. Eng. 1997, 17, 993-1003.

3. Perry, S.; Klemeš, J.; Bulatov, I. Integrating waste and renewable energy to reduce the carbon footprint of locally integrated energy sectors. Energy 2008, 33, 1489-1497.

4. Schuster, A.; Karellas, S.; Kakaras, E.; Spliethoff, H. Energetic and economic investigation of Organic Rankine Cycle applications. Appl. Therm. Eng. 2008, 29, 1809-1817.

5. Linnhoff, B. Pinch analysis - A state-of-the-art overview. Trans. Inst. Chem. Eng. Part A 1993, $71,503-522$.

6. Steam is sometimes used if industrial processes located outside of the site where the heat source is available are included in the heat distribution.

7. Shimoda, Y.; Nagota, T.; Isayama, N.; Mizuno, M. Verification of energy efficiency of district heating and cooling system by simulation considering design and operation parameters. Build. Environ. 2008, 43, 569-577. 
8. Critoph, R.E. Evaluation of alternative refrigerant-adsorbent pairs for refrigeration cycles. Appl. Therm. Eng. 1996, 16, 891-900.

9. Saljnikov, A.; Goričanec, D.; Kozić, Đ.; Krope, J.; Stipić, R. Borehole and Aquifer Thermal Energy Storage and Choice of Thermal Response Test Method. In Proceedings of the 4th WSEAS International Conference on Heat Transfer, Thermal Engineering and Environment, Crete Island, Greece, 21-23 August 2006; pp. 11-15.

10. Spurr, M.; Larsson, I. Integrating District Cooling with Combined Heat and Power, International Energy Agency (IEA); Programme of Research, Development and Demonstration on District Heating and Cooling; NOVEM: Sittard, The Netherlands, 1996.

(C) 2011 by the authors; licensee MDPI, Basel, Switzerland. This article is an open access article distributed under the terms and conditions of the Creative Commons Attribution license (http://creativecommons.org/licenses/by/3.0/). 